\title{
STUDY ON SUPPRESSION OF THE DYNAMIC BEHAVIOR OF COLLISION BULGE OF LEAD TUBE DRIVEN BY SLIDING DETONATION
}

\author{
LIU JUN, FU ZHENG, WANG PEI, YAO WEN \& QIAN JIAN-ZHEN \\ Institute of Applied Physics and Computational Mathematics, Beijing 100094, China
}

\begin{abstract}
When two head-on sliding detonation waves collide, some complex dynamical phenomena such as spallation and micro-jet always appear in the collision region of a metal tube, which cause its reliability to decrease. In the cylindrical implosion of lead/aluminum two layers tube, simulation results of the inner surface travel times of lead coincide well with the experimental results. In the polar position, there is a fracture cavity in the lead flyer, and a blunt bulge is formed on the inner surface. At the equator, large-scale fracture particles are generated as the inner surface of the lead flyer is growing. It is considered that the colliding bulge at the equator which seems to be continuous in the X-ray images is actually discontinuous, and it is composed of large-scale fracture particles and small-scale micro-jet particles. High-pressure Mach stem's fist impact in metal is considered as the main reason of the behavior of collision-bulging. And then, in order to avoid collision-bulging, high impedance metal iridium is used as a similar-rigid-control-block. Backward facing step model and regular reflection model are also designed to make the first shock wave intensity weakened in evidence in collision zone. Finally, the backward facing step model of lead and aluminum flyers driven by sliding detonation is simulated, and the influence of different length of model is analyzed. The simulation results show that the suppressing efficiency is enhanced to some extent while adjusting the length of control-block, and the suppression block does not affect the subsequent implosion process fly.
\end{abstract}

Keywords: detonation driven, colliding bulge, micro-jet, metal flyers.

\section{INTRODUCTION}

When two pairs of counter-slip detonation wave collisions drive the multi-metal flight layer, the outer surface of the fly-layer will experience a complicated loading and unloading state. The surface of the material has complex dynamic behaviors such as deformation protrusions, spallation failures and micro-jets. That is the slip detonation derived metal fly layer collision bump phenomenon studied in this paper. After the first shock wave in the metal flight layer collides, multiple incident and reflected waves are formed inside the flying layer-detonation product and the multi-flying layer, and the waves interact with each other again. The relationship between the collision area and the wave system is extremely complicated, the theoretical analysis of this problem is very difficult. At present, it is mainly addressed by experimental and numerical simulation studies.

According to the practical application of the specific problem needs, some scholars have designed experiments on two-point detonation driving metal fly layer collision bumps and fractures. In the study of the outward explosion problem, Singh et al. [1] observed the fracture time and fracture mode in the collision zone of the metal cylinder. Zhiembetov et al. [2] compared experimental results of cylindrical explosives of various metals; it was found that the physical properties of metal materials have a great influence on the bumping and crushing of the contact area. In the study of implosion problems, there is less research on metal flying layer bumps currently published. In 2013, Zhang et al. [4] carried out experiments on the explosion of two layers of aluminium and lead fly-core implosions that caused the detonation wave to detonate at two points, obtained experimental data on the 
development of spike-type bumps on the impact area and the micro-injection of the material field on the inner surface under implosion conditions. In 2015, Liu et al. [5] conducted a numerical simulation study on the cylindrical experiment. The analysis concluded that the high-pressure Mach rod loaded for the first time in the lead layer is the main factor in forming interface protrusions. Also, a trench-type micro-jet calculation model was designed, and it verified that the micro-jet at different positions in the interface of the lead flycatcher can be generated by the same uniform defect surface.

Detonation driven metal bumps introduce a series of additional problems. This phenomenon will reduce the reliability of the device and is undesirable in most cases. However, there is less research work on the suppression of this phenomenon. In the outward exopoise collision model, Zhang et al. [3] proposed adding a protective ring structure in the metal tube collision area to suppress the premature fracture in the collision area. Unlike the case of outward explosion, the dynamics of a multi-flying layer under implosion conditions are complicated. The introduction of a protection ring with reference to the outward explosion affects the polycentric compression of the fly layer. In this paper, based on the idea of impedance matching, shock backstep diffraction model and shock wave normal reflection model are designed to suppress the growth of collision bumps. The method of suppressing the impact bumping phenomenon is achieved by suppressing the formation of the high-pressure region of shock Mach in the lead layer, and the effectiveness of the method is verified by numerical simulation.

\section{SIMULATION OF IMPLOSION-DRIVEN EXPERIMENT}

Zhang et al. [4] used a cylindrical drum driven by explosive detonation flying lead and aluminum layer two motion implosion. Simultaneous detonation was applied from two pole positions in the experiment. Explosives detonation waves and internal shock waves in the fly layer collided at the equator. The plane model of this experiment and X-ray images and optical images are shown in Figs 1 and 2. The outer diameter of the explosive is $7 \mathrm{~cm}$ and the inner diameter is $5.4 \mathrm{~cm}$. The outer layer of the explosive is 304 steel shell and the thickness is $0.1 \mathrm{~cm}$. The explosives envelop two layers, the outer layer is $0.2 \mathrm{~cm}$ thick LY12 aluminium, and the inner layer is $0.25 \mathrm{~cm}$ thick pure lead.

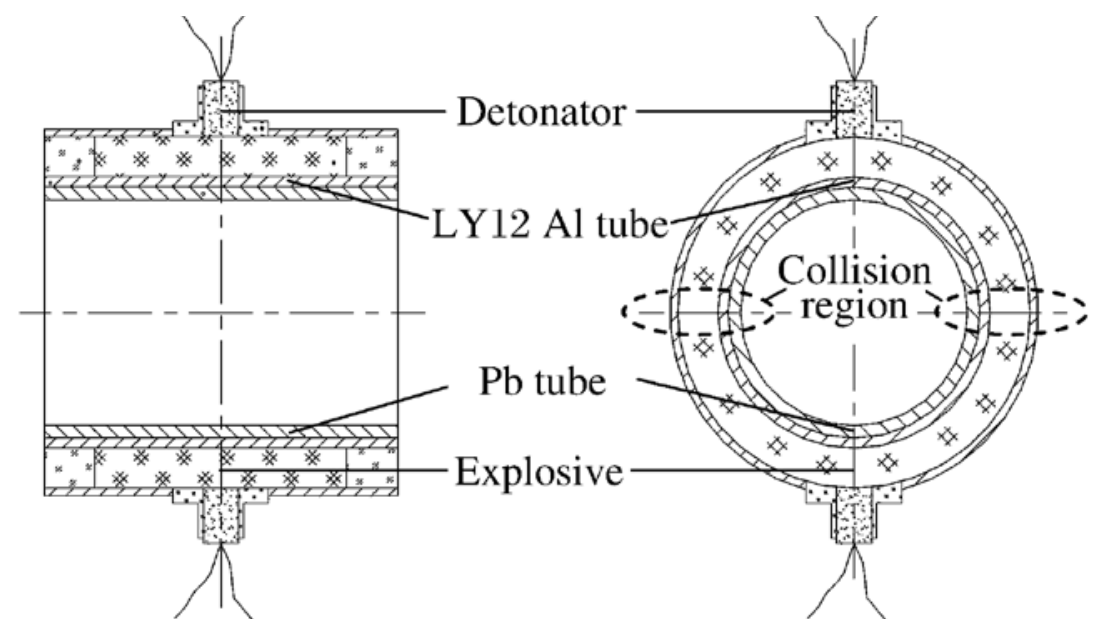

Figure 1: Schematic of experimental setup. 

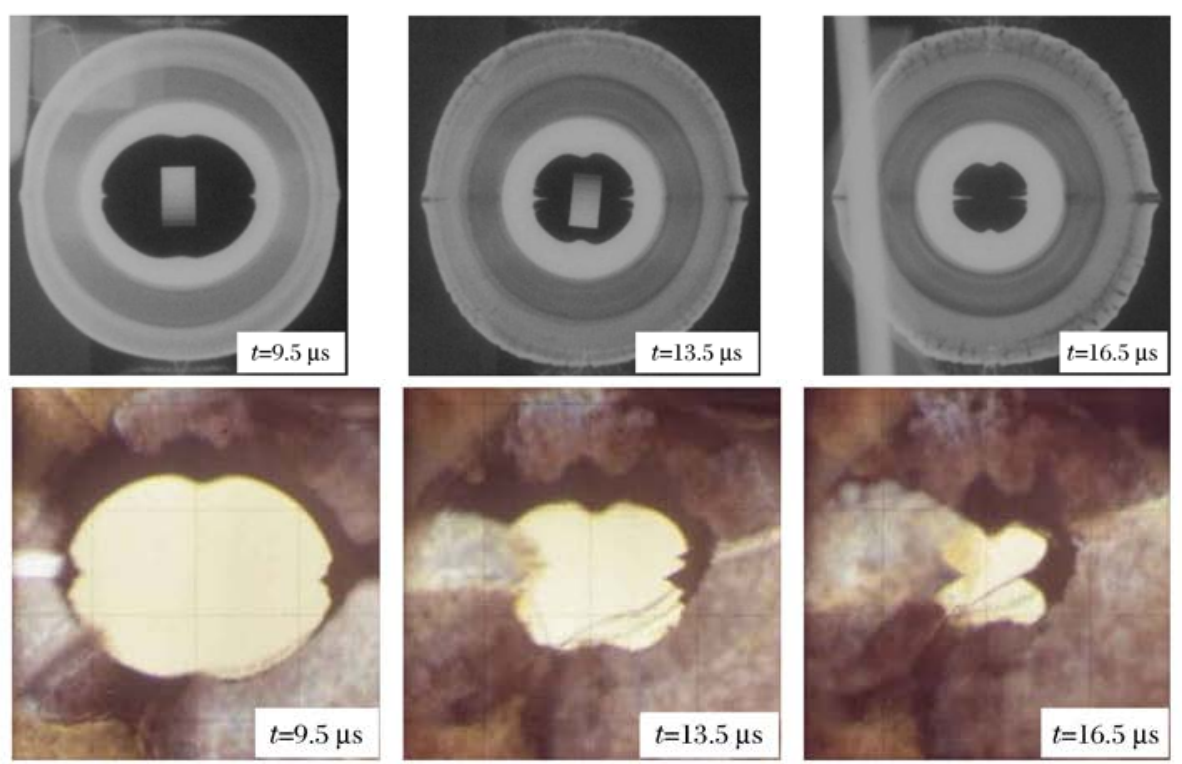

Figure 2: X-ray images and optical images of $\mathrm{Pb}$ tube driven by cylindrical implosion.

The Mie-Gruneisen equation of state for metals is:

$$
\mathrm{p}=\frac{\rho_{0} c^{2} \mu\left[1+\left(1-\frac{\gamma_{0}}{2}\right) \mu-\frac{a}{2} \mu^{2}\right]}{\left[1-\left(S_{1}-1\right) \mu-S_{2} \mu^{2} /(\mu+1)-S_{3} \mu^{3} /(\mu+1)^{2}\right]^{2}}+\left(\gamma_{0}+a \mu\right) E
$$

where

$$
\mu=\frac{\rho}{\rho_{0}}-1, E=e \rho_{0}
$$

For the explosives, the JWL equation of state is used:

$$
p=A\left(1-\frac{\omega}{R_{1} V}\right) e^{-R_{1} V}+B\left(1-\frac{\omega}{R_{2} V}\right) e^{-R_{2} V}+\frac{\omega E}{V}
$$

The specific calculation parameters are shown in Table 1.

Table 1: The JWL parameters of RX-06-AF.

\begin{tabular}{|c|c|c|c|c|c|c|c|c|}
\hline$\rho /\left(\mathrm{kg} / \mathrm{m}^{3}\right)$ & $P_{\mathrm{cj}} /(\mathrm{GPa})$ & $D /(\mathrm{m} / \mathrm{s})$ & $A /(\mathrm{GPa})$ & $B /(\mathrm{GPa})$ & $R_{1}$ & $R_{2}$ & $\omega$ & $E$ \\
\hline 1658 & 27 & 7800 & 503.03 & 9.065 & 4.3 & 1.1 & 0.35 & 7.6 \\
\hline
\end{tabular}


The calculation range is $[0,4.8] \times[0,4.8] \mathrm{cm}, \mathrm{dx}=0.006 \mathrm{~cm}$; the calculation result is compared with the experimental $\mathrm{x}$-ray image as shown in Fig. 3.

According to the numerical simulation, the cracks and the cavities are formed inside the lead flying layer at the two poles. As a result, bulge-like protrusions are formed on the inner surfaces of the two polar fly layers. Due to the tiny scale of the micro-jet particles generated on the inner surface of the two poles, neither the numerical simulation nor the X-ray images can reflect this phenomenon. The lead layer is sharper at the equator. The analysis shows that because some large-scale fractured particles fly at higher speeds, the X-ray image can capture the sharp protrusions at that location. However, due to the limitations of the numerical simulation grid size, some fractured particles that are similar to or smaller than the grid scale cannot be described. Therefore, the number of large-scale fractured particles in the collision zone in the experiment should be much larger than the numerical simulation results in this paper. It is believed that the lead material is not a continuum after the equator collision but breaks at the convex part to form many large-scale high-speed particles. Together with the small-scale particles formed by the micro-spraying, the lead material forms a bump phenomenon.

\section{SUPPRESSION METHOD ANALYSIS}

Under the sliding detonation driving condition, the first contact wave inside the lead layer will have a collision angle exceeding the normal reflection limit angle, thus forming a Mach reflection. Due to the higher pressure on the Mach rod moving toward the free surface, the lead flight layer forms a convex phenomenon that corresponds to the width of the Mach rod on the collision area. From a macroscopic point of view, this is due to the fact that the energy accumulation at the collision site causes more convexity. A first attempt was made to use a low-impedance energy-absorbing material to protect the collision area. The

Table 2: The parameters of metals [6].

\begin{tabular}{|c|c|c|c|c|c|c|c|c|c|}
\hline & $\rho_{0} /\left(\mathrm{g} / \mathrm{cm}^{3}\right)$ & $c_{0} /(\mathrm{km} / \mathrm{s})$ & $S_{1}$ & $\gamma_{0}$ & $\alpha$ & $G_{0} / \mathrm{GPa}$ & $Y_{0} / \mathrm{GPa}$ & $Y_{\max } / \mathrm{GPa}$ & $T_{\mathrm{mo}} / \mathrm{K}$ \\
\hline $\mathrm{Al}$ & 2.785 & 5.328 & 1.34 & 2.0 & 0.47 & 28.6 & 0.26 & 0.76 & 1222 \\
\hline $\mathrm{Pb}$ & 11.34 & 2.1 & 1.46 & 2.74 & 0.54 & 8.6 & 0.008 & 0.1 & 760 \\
\hline 304 Steel & 7.9 & 4.5 & 1.49 & 1.93 & 0.53 & 77 & 0.34 & 2.5 & 2380 \\
\hline
\end{tabular}
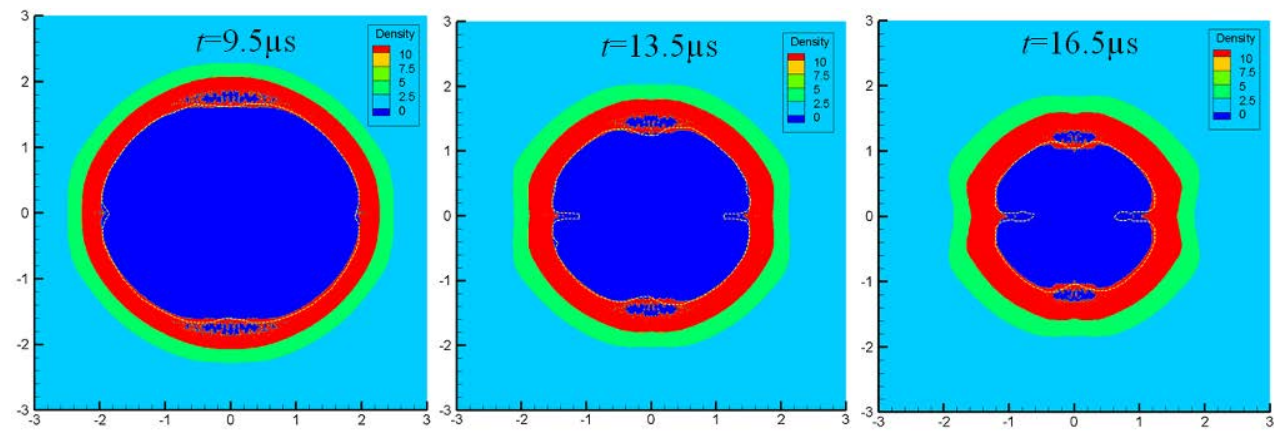

Figure 3: X-ray images, and density images by simulation, the yellow dashed line is the position of inner surface by $x$-ray image 
simulation can initially suppress the protrusion, but the low-impedance material compresses and bounces, causing a more serious bulge at the later stage of the simulation. Through the previous study [5], we found that the Mach rod with a certain width and high pressure that moves toward the free surface during the first loading of the lead flying layer is the main reason for the generation of the Mach rod's width. Therefore, in the following, the method of suppressing the contact bump is considered from the formation of the high-pressure Mach lever that suppresses the impact free surface, and the protective effect is mainly achieved by selecting a high-impedance material and modifying the detailed configuration of the contact portion. Two methods for suppressing projections are designed from two perspectives of sparse Mach rod pressure and suppression of Mach rod formation.

Method 1: Suppression of lead fly bulge by sparse Mach pressure. Due to the low strength of the lead material and the low melting point, the high-pressure Mach rod causes a high strain on the lead material after impact on the free surface of the lead fly layer, and even unloading and melting, head fragmentation and so on. If the pressure in the highpressure zone after the Mach rod can be reduced, it should be possible to effectively weaken the phenomenon of the lead fly bulge in the collision position. In the posterior problem of hydrodynamics, when the shock wave crosses the backstep, the sparse wave will be emitted outward from the singular point of the backstep and the shock wave diffraction effect will occur. This method is designed on this principle. Adding a high impedance step material to the contact area (Fig. 4), the pressure acting on the free surface is greatly reduced to achieve the purpose of reducing the deformation of the lead material by bumps.

Method 2: Suppression of lead scattering by forming regular reflections. In addition to the above-described method of suppressing the bulging of the sparse Mach rod high pressure region, a more direct method is to make the lead fly layer not to form a Mach reflection in the collision area. A wedge-shaped high-impedance material (Fig. 5) is added to the equator-impact region so that the impact angle of the first loading wave in the lead layer on the surface is smaller than the normal angle of reflection. So that the first loaded wave moves to the free surface in the form of a regular reflection.

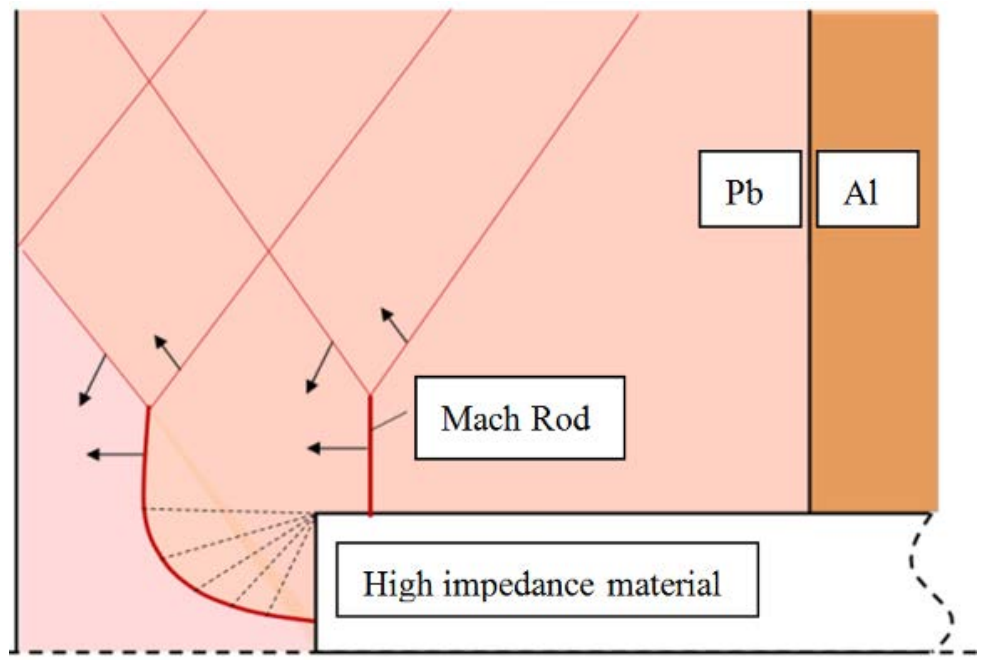

Figure 4: Rarefaction the pressure of Mach stem by shock wave diffraction. 


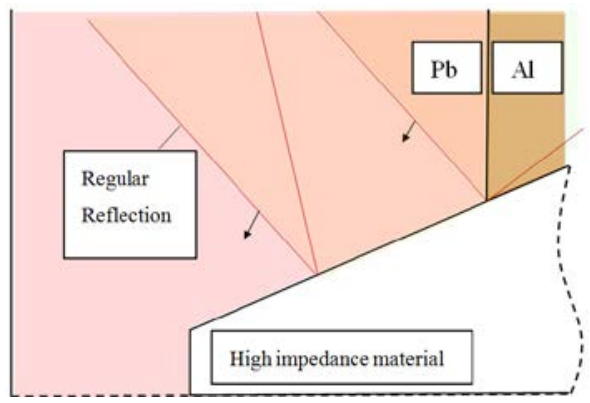

Figure 5: Shock wave in Lead forms a regular reflection by adding wedge block.

Theoretically, both method 1 and method 2 can reduce the pressure at the moment of the shock wave free surface, and thus to a certain extent, play a role in suppressing the collision phenomenon of the lead flight layer. The following numerical simulation method is used to test whether the above two methods can achieve the desired results without affecting the travel time and the centering effect of the inner layer of the flying layer.

\section{NUMERICAL SIMULATION OF SUPPRESSION METHOD FOR SLIDING DETONATION-DRIVEN PB FLY LAYER}

The above two methods of suppressing the bumps require the use of a high-impedance material, so that the multi-dielectric interface between the lead-high-impedance materials in the impact region is approximately fixed-walled. Here, we use metal iridium (Ir, a yellowwhite metal with high hardness, high elastic modulus, and highest density) as a highimpedance material for simulation studies. Compared to lead materials, germanium has a density about twice that of lead, an acoustic impedance that is four times that of lead and is not easily melted. The 2D plane calculation model is shown in Fig. 6. The protective design model for the collision of the original model is as follows.

Method 1: In the model where the projection is suppressed by the backstep diffraction, taking into consideration that the bump width is approximately 1.0 to $1.4 \mathrm{~mm}$, the thickness of the base metal is set to $1.6 \mathrm{~mm}$ and the length is $4.5 \mathrm{~mm}$, which is placed on the axis of the equator away from lead fly. Layer surface $1 \mathrm{~mm}$ position (Fig. 6(a)); method 2: In the model that suppresses bulges by forming regular reflections, the base metal isosceles trapezoidal body is $1.6 \mathrm{~mm}$ above, $6.8 \mathrm{~mm}$ below, and $4.5 \mathrm{~mm}$ above (Fig. 6(b)).
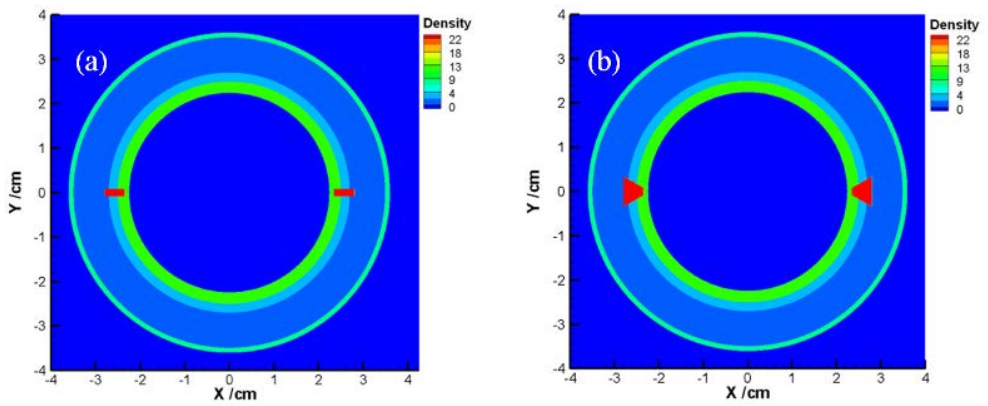

Figure 6: Two Suppression method. (a) Backward-facing step shock wave diffraction method 1; (b) Regular reflection method 2. 
Figs 7 and 8 show the results of the numerical simulation of the Method 1 and Method 2 for suppressing bumps. The red dashed line is the position of the internal interface at different times extracted from the experiment.

According to the results of numerical simulations, on the basis of the fact that the interfacial travel time in the lead layer is consistent with the experimental results, the two suppression projection methods analyzed in the previous section can all play a role in suppressing the bulging of the lead fly layer in the collision region. In addition, the tantalum material will disengage from the centering movement of the lead-aluminium fly layer after it plays a role in suppressing the convexity (the tantalum core velocity will be slower). After the tantalum material is detached, the lead and aluminium fly layer will be able to close smoothly and will not cause the tantalum to fly off. Layer fracture does not affect the subsequent centering of the fly layer.

For different real-world problem models, the size of the suppression block needs to be estimated a priori to achieve a better suppression effect. Here, the suppression method 1 is taken as an example to perform quantitative analysis of the inhibitory effect against the cylindrical experimental model mentioned in the previous section. The thickness of the rectangular block is still set to $1.6 \mathrm{~mm}$ and the length is set to variable. The tail overlaps with the outer surface of the aluminum fly layer $(\mathrm{R}=2.7 \mathrm{~cm})$, and the head position is calculated in 5 models with $\mathrm{R}=2.3,2.35,2.4,2.45$, and $2.475 \mathrm{~cm}$. The interfacial velocity in the lead fly layer within the angle of 0 to 15 degrees at the time of $16.5 \mu$ s was counted.

From the curve of Fig. 9, we can see that the interface velocity in the Pb-layer of the contact zone is the most gentle when the position of the head of the cubic block of the base metal is $2.4 \mathrm{~cm}$. This shows that the thickness of the block can be better adjusted by
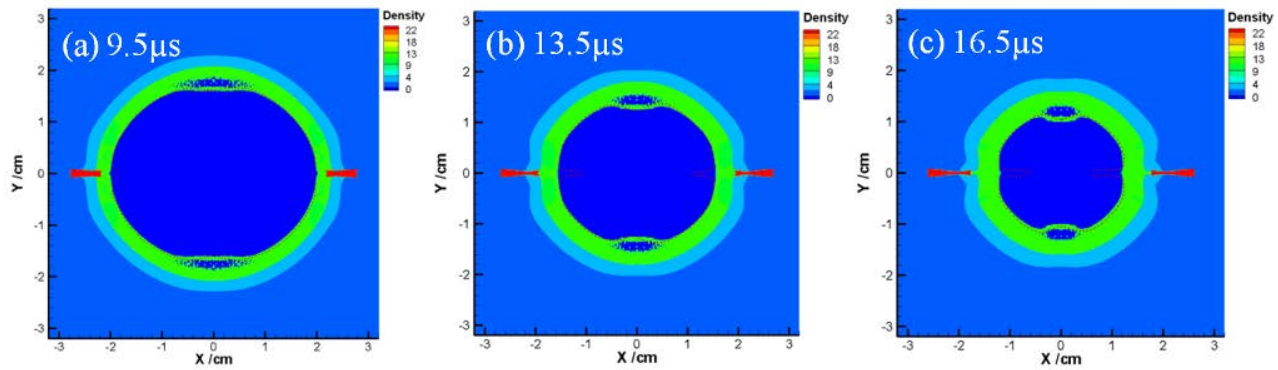

Figure 7: Density graph at different time by suppression method 1.
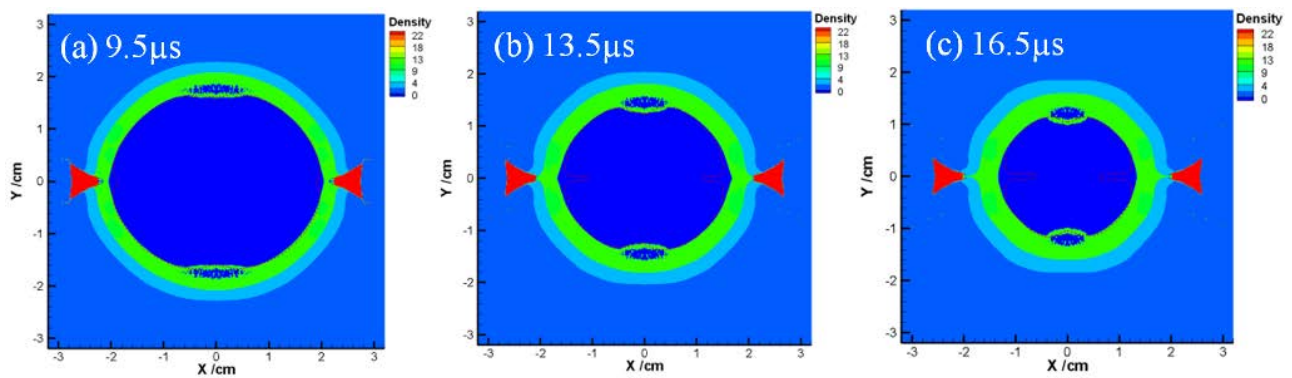

Figure 8: Density graph at different time by suppression method 2. 

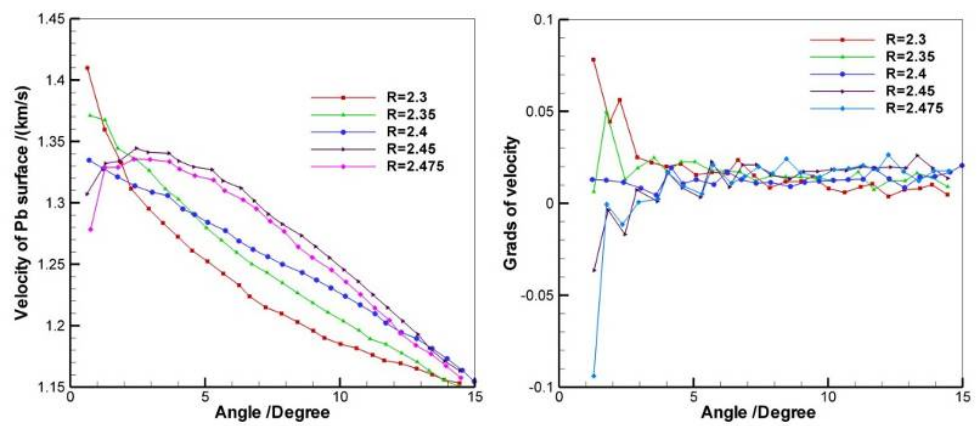

Figure 9: Velocity and gradient of inner surface of lead flyer in $0-15^{\circ}$ range, under the condition of different block length.

adjusting the length of the block. The same effect can be achieved by adjusting the thickness of the restraint block to achieve a better suppression effect.

\section{CONCLUSIONS}

In the study of the bumping problem of the metal flying layer driven by sliding detonation, two methods to suppress the bump of the metal flying layer are proposed in this paper: (1) the post step model of shock wave is constructed by adding a high impedance rectangular block to the position of the internal collision zone, and the high pressure of the Mach rod is sparse by the method of the shock wave diffraction of the back step to reduce the uplift deformation of the collision zone; (2) a high impedance wedge block is added to the collision area, which makes the first loading wave acting on its surface to form a normal reflection, reducing the high pressure area and reducing the bump.

The metal iridium is used as a high impedance block metal material, and the suppression effect of the two methods is tested by numerical simulation. The simulation results of the walk time in the lead layer can be in good agreement with the experiment, and the suppression results have not formed obvious large deformation in the free surface of the equatorial collision area. The iridium block is detached from the lead aluminium flying layer after completion of the block, and the lead flying layer can still maintain a continuous internal interface velocity during the subsequent process.

The suppression method proposed in this paper also has the following advantages. It can also be used for plane detonation to drive the fly layer collision model to suppress the collision bump phenomenon. After the material restraining block plays the role of inhibiting the protrusion, it will break away from the flying layer and will not affect the subsequent centering process of the flying layer. The problem introduced by using the suppression method of this paper is that the model parameters of the suppression block need to be simulated and a priori estimated for the different models, and the precision machining and detonation timing and the position control of the collision are brought into the experiment.

\section{ACKNOWLEDGEMENTS}

This paper is supported by the National Natural Science Foundation of China Youth Foud (11702028), and the National Defense Base Scientific Research Program (B1520132012). 


\section{REFERENCES}

[1] Singh, M., Suneja, H.R., Bola, M.S. \& Prakash, S., Dynamic tensile deformation and fracture of metal cylinders at high strain rates. International Journal of Impact Engineering, 27(9), pp. 939-954, 2002.

[2] Zhiembetov, A.K., Mikhaylov, A.L. \& Smirnov, G.S., Experimental study of explosive fragmentation of metals melts. Shock Compression of Condensed Matter, 620, pp. 547-552, 2002.

[3] Zhang, C.Y., Hu, H.B. \& Li, Q.Z., Experimental study on dynamic load behavior of plane lead-plane impinge region under detonation wave interaction. Chin. J. High Pres. Phys., 23(4), pp. 283-287, 2009.

[4] Zhang, C.Y., Hu, H.B. \& Li, Q.Z., Experimental study on dynamic load behavior of lead zone in impingement region under cylindrical implosion. Chin. J. High Pres. Phys., 27(6), pp. 884-888, 2013.

[5] Liu, J., Zheng, F., Feng, Q.J. \& Pei, W. Simulation study of the colliding bulge and surface micro-jet of metal flyers driven by detonation. Acta Physica Sinica, 64, pp. 234-701, 2015.

[6] Li, M.S. \& Chen, D.Q., Constitutive model of materials under high temperature and high pressure. Chin. J. High Pres. Phys, 15(1), pp. 23-31, 2001. 